\title{
The parameters of foam rolling, self-myofascial release treatment: a review of the literature
}

\author{
Przemysław Dębski ${ }^{1}$, Ewelina Białas ${ }^{1}$, Rafał Gnat ${ }^{2}$ \\ 1 Jerzy Kukuczka Academy of Physical Education, Katowice, Poland; ${ }^{2}$ Motion Analysis Laboratory, Department of \\ Physiotherapy, Jerzy Kukuczka Academy of Physical Education, Katowice, Poland
}

\section{Summary}

Self-myofascial release (SMR) is a well-known and popular therapy. Its growing popularity is based on high effectiveness and availability. However, there is a lack of agreement about which parameters should be used to optimize the effects of the therapy. The purpose of this review is to critically select and assess current literature and ascertain the values of the following parameters: (1) therapy duration, (2) volume of applied pressure, (3) speed and (4) frequency of roll, (5) type of roller, (6) the number of treatment applications during one session, (7) the duration of intervals between applications that yield the best results in terms of soft tissue.

The authors launched their research in May 2018. The search strategy included the electronic databases EBSCOhost and PubMed. The following inclusion criteria were assessed:

- English language, high quality manuscripts (evaluation in PEDro scale)

- at least one of the groups using the foam roller, tennis ball or the stick to fascial release

- basic parameters of therapy described.

A total 55 articles met the inclusion criteria. Patients can usually withstand a maximum tolerable pressure for 30-120 seconds, repeated 1-3 times, separated by 30 seconds of rest. The intensity of a single rolling movement should be moderate, and the movement should last about 3 seconds. Keeping the roller on particularly sensitive areas is recommended to release tension and enhance blood perfusion.

Currently, there is no consensus on an optimal FR programme. However, there is a tendency to use SMR tools with a physiology-based method to enhance therapeutic efficiency.

\section{Keywords: Self-myofascial release - Foam rolling - Connective tissue - Fascia - Therapy}

\section{Introduction}

Self-myofascial release (SMR) is a collective term for manual therapy techniques based on the effects of applying mechanical force to soft body tissue [55]. Self-myofascial release is widely used by people who are physically active, is one of the tools used by physiotherapists in their work with patients, and is useful for athletes from all sporting disciplines at all levels of competition [14, 42]. The main objective of adding SMR to a set of therapeutic techniques or to training aids is improvement in range of motion (ROM) and a reduction in post-training muscular pain [21, 68].

Although rollers of various density are used increasingly in SMR, many authors point out that there is still a lack of robust scientific evidence documenting their effects on the fascia in the literature [71]. Furthermore, a lack of agreement on the values of basic therapeutic parameters is very apparent: the duration of treatment and of breaks, the type of roller to be used, the force to be applied on the tissue, the speed and frequency of rolling, and also the number of times the treatment should be repeated in one session [14, $57,65]$. As the literature contains few details on the methods of treatment, it is more difficult to establish a theoretical estimate of the therapeutic benefits of SMR. It could be said that some of the descriptions of the benefits of this kind of therapy are anecdotal in nature, and demand a much more methodologically precise approach to the topic [66]. This issue is even more significant these days, as SMR has been successfully adapted to the needs of the fitness market and physiotherapy [67]. As a result, the tools used in SMR have become accessible for the domestic user with 
little experience, limited awareness of their body, and a lack of basic knowledge of the way the treatment works, and also for people who treat patients with symptoms of musculoskeletal system dysfunction [61, 67].

The basics of the way SMR affects the body are similar to the mechanisms that are activated during self-massage, and these terms are often used interchangeably. This also applies to the forms of SMR which use rollers (FR - foam rolling). The rollers are used to lengthen and apply pressure to the fascia, which in turn stimulates histological tissue changes in the area being treated, which has undergone pathological changes through strain, traumatising movements, metabolic dysfunction and even psychological factors [32, 42]. Self-treatment is supposed to lead to the elimination of symptoms known as fascial restrictions and adhesions, such as pain and decreased ROM $[34,68]$.

There are many theories which attempt to explain the effects obtained during myofascial release, and which also occur during foam rolling therapy. These effects include: changes in elasticity caused by the thixotropic properties of soft tissue [37, 44, 65, 68], a piezoelectric effect [9, 55] release of fascial adhesions $[51,55]$ cellular response to pressure $[9,55]$, stimulation of tissue fluid flow $[9,44]$, neural inhibition [68] and release of trigger points [43, 55, $66,77]$. It is worth emphasizing that the authors often do not limit their work to the identification of one of the mechanisms above, but rather give two or three of them, which are related to each other logically. As an example, the increase of tissue temperature by friction will surely lead to thixotropic changes, and, as a result, easier release of adhesions. Similarly, increased blood flow to the tissue will lead to oxygenation and may put an end to the energetic crisis which is referred to as one reason behind the formation of trigger points [74].

There is no lack of evidence for the effectiveness of self-myofascial release. It is worth emphasizing that the results of treatments carried out differ from each other significantly. One of the principal factors which might have an effect on this significant variation is an unspecified research methodology, and in particular, the basic parameters of treatment: time, intensity and speed of rolling, length of pause between each set of rolling, and also the number of rolling sets during one self-treatment session.

For example, Vigotsky et al. [71], MacDonald et al. [37] and Markovic [41] used two sets of self-release for 60 seconds during one treatment session, Kelly and Beardsley [34], Peacock et al. [56] and Skarabot et al. [65] obtained statistically significant effects from 30 -second treatments, and Sullivan et al. [68] used 5- and 10-second sets as appropriate. Such large differences in significant treatment parameters make the evaluation of SMR as a generally accessible and widely used treatment method difficult. Very important outcomes were observed in the study by Bradbury-Squires et al. [4]. They used the same protocol of rolling in 5 repetitions but in one group 20 seconds of rolling was recommended whereas in the other group it was 60 seconds. A 10\% increase in knee ROM was observed in the first group and a $16 \%$ increase was observed in the group with longer treatment.

Regarding the force applied, there arises the question whether greater pressure exerted on tissues provides greater therapeutic effects. Aboodarda et al. [1] described high muscle vertical pressure as a $1-3 \mathrm{~cm}$ deep muscle therapy, but they obtained this outcome with a hard rubber roller massager and the command not to produce a greater value than $7 / 10$ on the Visual Analogue Scale. There is a tendency to use a command such as AMAP - as much pressure as possible - and AMAT - as much pressure as tolerable. Kelly and Beardsley [34] suggested, based on Schleip's work, that the force applied during rolling may serve to increase parasympathetic nervous activity through the stimulation of mechanoreceptors. However, a key issue for this parameter is perception of pain. Pain is a very complex multidimensional process involving the central nervous system and other systems of the body [13] that can occur if too much pressure is used. It is also suggested that higher density tools may have a stronger effect than softer density [15].

Another parameter that requires discussion is frequency of rolling determined by the number of repetitions and cadence of motion. This parameter can play the key role in improving fluid flow in tissues, which is described as one of the mechanisms responsible for changes in range of motions [44] as well as the often mentioned release of fibrous adhesions between the different layers of the fascia [68]. There is a possibility that researchers could obtain a different result if they applied a fixed cadence and a fixed pressure. Also it is hypothesized that changes in fascia are caused to some extent by friction resulting from rolling [68].

Among the most important parameters are the number of sets during one treatment session and the duration of pause maintained between sets. There is a lack of agreement how many times the application should be repeated to maximize positive outcomes. For practical purposes it should be determined whether an additional set of rolling could possibly bring more valuable results. The potential association between volume of the therapy and the results was first suggested by Skarabot and Beardsley [65], because they suspected that greater duration of treatment was responsible for their results.

The aim of the study was to compare the treatment parameters used up to now by researchers and then, on the basis of the data collected, to answer the following question:

Which parameter value - duration of treatment, level of force applied to tissue, type of therapeutic tool, speed and frequency of rolling, and also number of applications of treatment during one treatment session and duration of 
the break between these applications - demonstrates the greatest effect on soft tissue in the current literature?

\section{Material and methods}

The authors divided the evaluation into several stages based on the PRISMA search strategy [40]. Firstly, abstracts and titles were identified through a database search. The desired research papers had to contain the key words "self-myofascial release" or "foam rolling". The search engines EBSCOhost, Google Scholar and PubMed were used for this purpose. Only papers in English were included.

\section{SMR: 261 items}

FR: 326 items (as of 25.10.2018)

Secondly, duplicates and commercial items were rejected. Then, remaining articles were screened through inclusion criteria and assessed for eligibility. The remaining criteria for inclusion were:
- the use of a cylindrical roller, tennis ball or a hand roller for massage in the treatment

- a description of the basic treatment parameters

- a PEDro scale evaluation of the research $[40,50,52]$ of 6 or higher

- the research compared an FR group with another FR/ SMR group, another treatment technique and/or a control group.

In the last stage, the remaining articles were included in the synthesis.

\section{Results}

Fifty-five of the 587 papers analysed met the criteria for inclusion. This result suggests that there is not much high quality research on the topic of rolling in the available literature. Most research in the databases consists of overviews or commercial research, generally with no scientific merit. After the initial selection, the next stage involved evaluating the articles based on their research

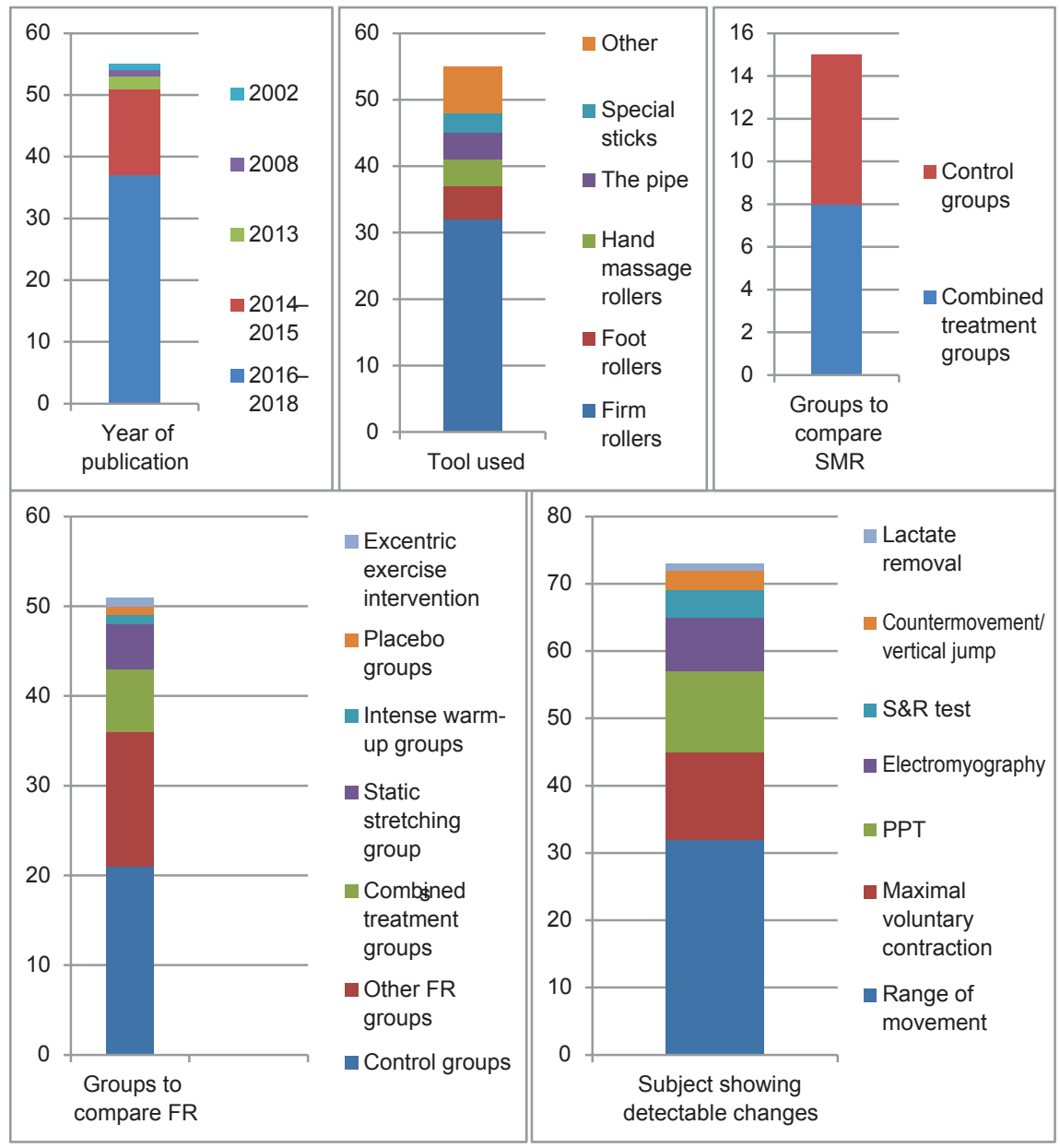

Fig. 1. Data obtained from the articles included into the review. SMR - Self Myofascial Release, FR - Foam Rolling, PPT Pressure Pain Threshold, S\&R - Sit and Reach test. In the „groups to compare” graphics, presented is the data describing groups to which the baseline therapy (SMR or FR) was compared 
methodology using the PEDro scale. Many papers were rejected at this stage as well. The average PEDro score for the selected papers was 6.4. When the article was given a high PEDro score, but the authors did not include information on the treatment parameters used, the paper was also excluded from this literature review. The basic data of papers included in the analysis are presented in Figure 1.

Most of the research papers described are from 2014 onwards ( 51 papers $=93 \%$ ), of which as many as 37 papers are from 2016-2018 (67\%). The older works date from 2002 (1), 2008 (1), and 2013 (2). These statistics are evidence of the growing popularity of the topic of myofascial release using rollers, and that knowledge about the usefulness of this treatment has still not been systematized.

In 32 selected papers, the authors decided to use a foam roller. Firm rollers made up 58\% of the equipment used in the research. Four authors (7\%) chose the more comfortable version of the roller (the pipe), which is long and made of softer materials. Another four authors (7\%) used hand roller massagers, while three $(6 \%)$ used a special stick to roll the soft tissue. In five papers (9\%) different balls were used (in each case, this was due to the paper being on the release of the sole of the foot). In this last case, the authors consistently used the term SMR rather than FR.

The groups used to compare results with the FR groups were most often control groups (21 papers), other FR groups (15), static stretching groups (5), combined treatment groups (7), intense warm up (1) or a placebo (1).

In the set of SMR articles (papers in which tennis balls were used for rolling), the results were compared to control groups (7). In eight papers, rolling was used in combined treatment. For example, in one of them, an SS (static stretching) group was compared with an FR + SS group, while in another paper, groups which rolled using a roller and a tennis ball were given the same treatment, but with different application times [46]. The most common nomenclature is described in Figure 2.

In the clear majority of papers, statistical differences between groups were sought mainly in the ROM - range of motion ( 32 papers $-58 \%$ ). Among the many remaining parameters used to compare the results of SMR therapy, the most common were MVC (maximal voluntary contraction $-24 \%)$, pain pressure threshold (22\%) and EMG parameters (electromyography $-15 \%$ ).

In the papers included in this article, the most common time values for a single rolling period were 30,60 and 120 seconds. The authors usually choose "as much pressure as possible", or the subjective level of the participant's pain felt during therapy to determine the level of force applied to the roller. The authors evaluated the value of the force applied to the tissue in the case of applying the whole body to the roller. In the case of the hamstrings group, depending on the place of measurement on the thigh, the percentage amounts for the role of the whole body mass ranged from $25.44 \pm 3.86 \%$ by the knee joint to $46.44 \pm 4.7 \%$ by the hip joint [57]. The most commonly used number of repetitions was one, two or three rolling sets during a treatment session, separated usually with a 30 -second break or shorter. The authors noted the type of roller used in the studies. It was found that harder rollers are more effective in achieving myofascial release [15]. Basic parameters of papers included in the review are presented in Table 1.

In the interests of greater transparency, the authors have decided to analyse the individual parameters in separate paragraphs.

\section{Type of roller}

Curran et al. [15] dedicated all of their paper to comparing two kinds of roller available on the market: the hard version (MRR - Multilevel Rigid Roller), and the

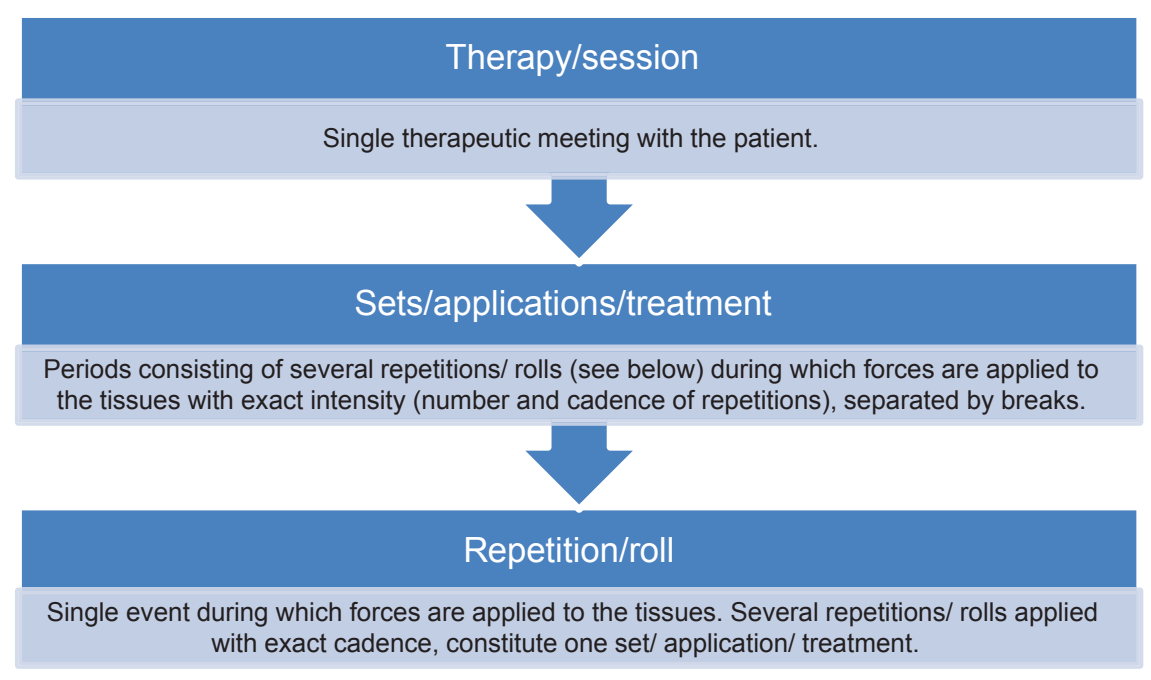

Fig. 2. Due to the discrepancies in the literature associated with the course of therapeutic intervention, the most common terminology was presented and explained to what the definitions apply 
Table 1. Basic analysis of papers included to review. The most common parameters and methods used in papers has been presented. Shortcuts: AMAT - As Much As Tolerable, AMAP - As Much As Possible, TBW - (Subject's) Total Body Weight, VAS - Visual Analogue Scale

\begin{tabular}{|c|c|c|c|}
\hline Parameter & Most common methodology & $\begin{array}{l}\text { Quantity of most common } \\
\text { parameters/methods }\end{array}$ & $\begin{array}{c}\text { Quantity of positive } \\
\text { outcome }\end{array}$ \\
\hline \multirow{6}{*}{ Type of roller } & Firm rollers & $32(58 \%)$ & 25 \\
\hline & Foot rollers & $5(9 \%)$ & 3 \\
\hline & Hand roller massagers & $4(7 \%)$ & 4 \\
\hline & The pipe & $4(7 \%)$ & 4 \\
\hline & Special sticks/poles & $3(6 \%)$ & 3 \\
\hline & Other & $7(13 \%)$ & - \\
\hline \multirow{5}{*}{ Force of rolling } & AMAT & $2(4 \%)$ & 2 \\
\hline & AMAP & $16(29 \%)$ & 15 \\
\hline & TBW & $2(4 \%)$ & 2 \\
\hline & VAS scale $7 / 10$ & $6(10 \%)$ & 3 \\
\hline & Other & $29(53 \%)$ & - \\
\hline \multirow{4}{*}{ Duration of application } & $30 \mathrm{sec}$ & $17(31 \%)$ & 15 \\
\hline & $60 \mathrm{sec}$ & $18(33 \%)$ & 15 \\
\hline & $120 \mathrm{sec}$ & $14(25 \%)$ & 10 \\
\hline & Other & $6(11 \%)$ & - \\
\hline \multirow{3}{*}{$\begin{array}{l}\text { Number of applications in } \\
\text { one treatment session }\end{array}$} & Single & $26(47 \%)$ & 24 \\
\hline & Three repetitions & $16(29 \%)$ & 13 \\
\hline & Other & $13(24 \%)$ & - \\
\hline \multirow{4}{*}{$\begin{array}{l}\text { Number of a treatment } \\
\text { sessions }\end{array}$} & One session & $41(74 \%)$ & 33 \\
\hline & Three sessions & $5(9 \%)$ & 5 \\
\hline & More than three sessions & $7(13 \%)$ & 6 \\
\hline & Other & $2(4 \%)$ & - \\
\hline \multirow{3}{*}{ Intensity of rolling } & Short time $(1-3 \mathrm{sec})$ & $18(33 \%)$ & 16 \\
\hline & Long time $(3 \mathrm{sec}+)$ & $9(16 \%)$ & 7 \\
\hline & Other & $28(51 \%)$ & - \\
\hline \multirow{4}{*}{ Length of break } & No break & $5(9 \%)$ & 4 \\
\hline & Up to $30 \mathrm{sec}$ & $18(33 \%)$ & 17 \\
\hline & $60 \mathrm{sec}$ & $4(7 \%)$ & 2 \\
\hline & Other & $28(51 \%)$ & - \\
\hline
\end{tabular}

soft version (BFR - Bio-Foam Roller). The researchers found that a statistically significantly stronger effect occurred with the hard roller during three rolling periods on the side of the thigh (the values expressed in $\mathrm{kPa}$ were almost twice as high). Also Cheatham and Stull [12] used different types of roller. For range of motion they found no statistically significant differences between medium, hard (both increased passive knee ROM by $8^{\circ}$ ) and soft density roller (increased ROM by $7^{\circ}$ ). Similar results were observed in pressure pain thresholds (PPT). There was a post-intervention increase of $180 \mathrm{kPa}(\mathrm{p}<0.001)$ for the medium density roller, $175 \mathrm{kPa}(\mathrm{p}<0.001)$ for the soft density roller, and $151 \mathrm{kPa}(\mathrm{p}<0.001)$ for the hard density roller. Referring to the existing literature, the researchers expressed the opinion that working on a given tissue with greater force brings about a deeper, and so more thorough, penetration of the tissue, more intensive stimulation of the autonomic nervous system and a greater resulting relaxation, when compared to the softer equivalent [15]. Other authors note that it is best to assign conventional rollers to inexperienced users, whilst if the application of a larger dose of treatment is required, hard rollers should be used 
[66]. Bradbury-Squires et al. [4] and Sullivan et al. [68] used programmed devices in their research, which on the one hand allowed for the force used on the whole length of the myofascial unit being treated to be uniform, while on the other hand it reduced the practical value of the research. In the case of treating difficult-to-reach surfaces of small surface area, such as the sole of the foot, the use of tools of an appropriate size appears to be justified. Different types of ball (tennis, golf, lacrosse) are tools of this sort, and were used by some authors in their research [20, 25, 45, 55, 69]. Macdonald et al. [37] used a polyvinyl chloride pipe wrapped in $1 \mathrm{~cm}$ thick neoprene foam in their research. In this way, it was possible to limit the contact area of the roller with the body, and so increase the application force on the areas being treated, while maintaining a fairly high level of firmness. SMR carried out with the constructed roller produced statistically significant changes in range of movement, of $4^{\circ}$ to $20^{\circ} 2$ minutes after, and $3-17^{\circ} 10$ minutes after treatment [37]. This confirms the theory that release can take place superficially, but a therapeutically significant effect demands work with a greater force on deeper tissue $[17,65]$. De Bruyne et al. [17] point to the greater therapeutic value of the hand roller, compared to the classic cylindrical foam roller. Monteiro et al. [45] came to the opposite conclusion, noting better results in each foam roller group compared to the equivalent hand roller groups, but only the group with 120 -second treatment showed a statistically significant difference compared to its 60 -second equivalent from the hand roller group.

\section{Force of application}

The instructions on how strongly the research subjects should be applied to the roller can be organized into two groups. The first of these is application with the greatest possible force (AMAP - as much as possible), and the second option is working with pressure not causing more discomfort than 7 on a 10-point scale. Few studies used total body weight (TBW) as a basic parameter but it probably means that participants tried to place their entire weight at the place of contact with the roller. In clinical treatment of trigger points, pressure is applied up until the first moment at which resistance turns into pain, and application is maintained until the sensation is removed or significantly lessened, and then the new boundary between pain and discomfort is sought [58]. Pearcey et al. [57] were first to use the concept of taking the subjective sensations of the patient as the upper limit of treatment, marking out the tolerance of the research subject (AMAT - as much as tolerable). Bradbury-Squires et al. [4] suggested force amounting to $25 \%$ of the body weight of the patient, while Sullivan et al. [68] stood out by suggesting a uniform force of application of $13 \mathrm{~kg}$ for all research participants. This kind of approach helps to unify the methodological procedure, but, bearing in mind the varied morphology (and personality) of patients, it is justified only if the research group is very uniform. During self-massage with a roller with one's own body weight (bearing one's weight only on the contact point with the roller), it is difficult to achieve any greater pressure, meaning that the TBW and AMAP values are close or even identical. For the same reason, in order to reduce tension and pain at a particularly sensitive point when rolling with constant pressure, keeping the roller in the place where increased discomfort is felt is recommended. It is worth emphasizing that rolling itself, like sports massage, can lead to pain, micro-bleeding and inflammation, which can last for up to 24 hours [58].

In the light of the benefits of applying pressure to tissue, the question should be posed: is the curve representing the relationship between the effect of treatment and the force of treatment directly proportional, or rather up to what point is this the case? In other words, to what extent will greater pressure on the tissue be reflected in a greater range of movement without damage to the tissue?

The authors suggest that directed pressure combined with tensing or relaxing muscles can shape the length of the sarcomeres and number of them (myofibrillogenesis), reducing pain as a result $[64,74]$. However, this is only a scientific theory.

\section{Duration of application}

The times most commonly used by the authors were 30,60 and 120 seconds. This is probably due to the fact that manual techniques for fascial work with patients fit into these time intervals [68]. However, none of the authors specified which intervals were effective for manual self-release techniques $[4,68]$. The second reason behind these time values is certainly the fact that comparisons of the therapeutic effect of FR/SMR and stretching are often made $[20,34,35,44,50,62,65,75]$. Macdonald et al. [37] suggest using an application time of between 60 and 90 seconds. Patel et al. [55] lean towards the statement that most research subjects obtain a therapeutic effect in a time period that ranges between 60 and 120 seconds of treatment. In opposition to this hypothesis, Couture et al. [14] stated that a time period of less than 2 minutes is not sufficient to obtain improvement in the range of movement in the knee joint. Vigotsky et al. [71] and MacDonald et al. [37] used two repetitions of 60 seconds in their studies. Bushell et al. [5] used 60 seconds of rolling but repeated this more frequently, both in terms of number of applications and number of treatment sessions. In these cases, statistically significant differences in the parameters under examination were achieved compared to the control group. In shorter times, a few authors also obtained positive results $[24,27,65]$. In recent studies there is a tendency to roll a few groups of muscles called "regions", one after another $[54,69,31,22,35,39]$. Also Cheatham et al. divided 
the quadriceps muscle into two zones [8,11]. Despite the short duration of application of one muscle group, positive outcomes often occur. It may be caused by adding up benefits of therapy of a few regions that are lying close to each other. Especially the methodology described by Madoni et al. [39] is worth citing. The authors divided hamstring groups into three sections. Every application lasts for 10 seconds on one section. Myofascial release of all three sections ( 10 seconds per section, 30 seconds in total) was followed by a 10 -second break. After that, the whole set of rolling was repeated two more times. Very short duration of application in one section (30 seconds divided into three sets) was enough to achieve a positive result.

In the context of this issue, the study by Sullivan et al. [68] is very valuable, as the number of repetitions and their duration were varied while the pressure and frequency of rolling remained the same. An increase in range of movement in the sit and reach test of $4.3 \%$ was observed in subjects who rolled for 10 seconds, compared to those who rolled for 5 seconds. The authors themselves point out that this is not sufficient to draw conclusions, and that there is a need to study the relationship between length and intensity of treatment and effects obtained. Monteiro et al. [46] also chose to compare the effects of treatment of different durations. Their analysis indicates that treatments lasting above 90 seconds produce better effects in improvement of FMS (functional movement screen) "overhead deep squat" than treatments lasting less than 90 seconds.

The results indicate that there is a relationship between a greater duration of application and greater secretion of cytokine and growth factor [6].

\section{Number of applications in one treatment session}

In terms of the number of rolling periods in one session, the studies can be divided into two groups. The first is made up of studies in which the authors used a single rolling set $[8,10,16,19,22,24,29,33,38,45,53,55,57$, $59,68,69,73]$. In the other studies, the values $2,3,4$ and 5 appeared with similar frequency, with three rolling sets being the most commonly chosen option $[3,5,15,23,26$, $35,39,46,57,62]$.

\section{Number of treatment sessions}

As many as 41 authors carried out their studies in one treatment session. In the remaining studies, two $[47,48,68]$ or three $[15,33,37,38,57]$ treatment sessions were used as well. Seven researchers used a longer protocol. Six out of these noted statistically significant differences in their treatments $[3,5,31,44,47]$. This is not necessarily the result of the additive effects of subsequent sessions, since many other authors reached statistical significance despite carrying out only one session. The significance of the number of treatment sessions parameter loses its significance in the context of the study by Kelly and Beardsley
[34], who noted a tendency for the effects of FR therapy to be maintained only for up to 20 minutes.

\section{Intensity of rolling}

Due to their properties, fascia do not respond to short, quick stimuli at high amplitude [70]. Their thixotropic properties make them reminiscent of plasticine: when shaped with appropriate force for a specified period of time they change their structure significantly. As a result, the use of techniques that affect the fascia with low speed appears to be justified. A classic example of a slow effect on soft tissue is the widely used method of deep tissue massage, in which the therapist reduces friction to a minimum.

The relatively slow rolling technique with a moderate cadence is also attractive due to the more effective flow of fluids which are drawn mechanically by force of application. Directed pressure through slow rolling and a fluid flow appear to be justified physiologically.

Despite the scientific theories in $30 \%$ of studies the authors who described this parameter in their research methodology used dynamic, one - to three-second movements $[7,14,22,23,24,26,27,31,35,38,44,53,54,56,62]$. This means that the pressure from the roller moved through the space from the beginning to the end of the treated area in this time. Curran et al. [15] were one of the exceptions to the rule, using 10-second repetitions when studying the difference between rollers of various levels of hardness. D'amico \& Paolone [16] suggested a length of repetition of 5 seconds. They did not obtain statistically significant differences in their research.

Morton et al. [51] suggested an entirely different methodology, comparing the results of static stretching with the same therapy preceded by four 60 -second repetitions of rolling. This long period of time turned out to be entirely ineffective, and no statistically significant differences were found between the basic treatment and the treatment enriched by SMR.

\section{Length of break}

The break between sessions in the studies usually amounted up to 30 seconds (33\% of studies). This short break is due to the specific nature of the treatment, which is associated with fast, short-term results [34, 65]. Additionally, it should be remembered that rolling is often not an end in itself, but precedes or rounds off a training session or a sporting competition. For this reason, competitors cannot spend a long time rolling.

\section{Summary and conclusion}

The authors of the selected studies vary in terms of methods they propose for myofascial release using a hand roller or conventional roller. Nevertheless, the majority 
use parameter values which are justified from a physiological point of view. Using the treatment parameter values described here does not guarantee therapeutic success, but may be sufficient to obtain satisfactory results. In the future, it will be necessary to specify what the mechanisms of the application of myofascial release tools on soft tissue are, in order make it easier to create a more precise treatment programme, both for FR therapy and for all treatments such as myofascial release.

The most desirable forms of treatment in terms of therapeutic effect are treatments using a firmer type of roller, with as great a force of application as possible (as tolerated by the patient) for a period of 30-120 seconds. The intensity of a single rolling movement should be moderate, and the movement should last about 3 seconds. Keeping the roller on particularly sensitive areas is recommended. During the course of one session, it is worth using between 1 and 3 applications, separated by a 30 -second break. If deeper therapeutic penetration of the tissue is needed, a firmer version of the roller is recommended.

\section{Conflict of interest: Authors state no conflict of interest.}

\section{References}

1. Aboodarda S.J., Greene R.M., Philpott D.T., Jaswal R.S., Millet G.Y., Behm D.G. (2017) The effect of rolling massage on the excitability of the corticospinal pathway. Appl. Physiol. Nutr. Metab., 43(4): 317-323. DOI: 10.1139/ apnm-2017-0408.

2. Aboodarda S.J., Spence A.J., Button D.C. (2015) Pain pressure threshold of a muscle tender spot increases following local and non-local rolling massage. BMC Musculoskeletal Disorders, 16(295). DOI: 10.1186/s12891-015-0729-5.

3. Bishop C., Aune A.A.G., Turner A.N., Papadopulos K., Budd S., Richardson M., Maloney S.J. (2018) Acute and chronic effects of foam rolling vs eccentric exercise on ROM and force output of the plantar flexors. DOI: 10.1080/02640414.2018.1486000.

4. Bradbury-Squires D.J., Noftall J.C., Sullivan K.M., Behm D.G., Power K.E., Button D.C. (2015) Roller-massager application to the quadriceps and knee-joint range of motion and neuromuscular efficiency during a lunge. J. Ath. Train, 50(2): 133-140.

5. Bushell J.E., Dawson S.M., Webster M.M. (2015) Clinical relevance of foam rolling on hip extension angle in a functional lunge position. J. Strength Cond. Res., 29(9): 2397-2403.

6. Cao T., Hicks M., Campbell D., Standley P. (2013) Dosed myofascial release in three-dimensional bioengineered tendons: effects on human fibroblast hyperplasia, hypertrophy and cytokine secretion. J. Manipulative Physiol. Ther., 36(8): 513-521.
7. Casanova N., Reis J.F., Vaz J.R., Machado R., Mendes B., Button D.C., Pezarat-Correia P., Freitas S.R. (2017) Effects of roller massager on muscle recovery after exercise-induced muscle damage. J. Sports Sci., DOI: 10.1080/02640414.2017.1280609.

8. Cheatham S.W., Baker R. (2017) Differences in pressure pain threshold among men and women after foam rolling. Journal of Bodywork \& Movement Therapies 21: 978-982. DOI: 10.1016/j.jbmt.2017.06.006.

9. Cheatham S.W., Kolber M., Cain M., Lee M. (2015) The effects of self-myofascial release using a foam roll or roller massager on joint range of motion, muscle recovery and performance: a systematic review. Int. J. Sports Phys. Ther., 10(6): 827-838.

10. Cheatham S.W., Kolber M.J., Cain M. (2017) Comparison of video-guided, live instructed, and self-guided foam roll interventions on knee joint range of motion and pressure pain threshold: a randomized controlled trial. Int. J. Sports Phys. Ther., 12(2): 242-249.

11. Cheatham S.W., Stull K.R. (2018) Comparison of a foam rolling session with active joint motion and without joint motion: A randomized controlled trial. Journal of Bodywork \& Movement Therapies, 22(3): 707-712. DOI: 10.1016/j.jbmt.2018.01.011.

12. Cheatham S.W, Stull K.R., Kolber M.J. (2018) Comparison of a vibration roller and a nonvibration roller intervention on knee range of motion and pressure pain threshold: a randomized controlled trial. DOI: 10.1123/ jsr.2017-0164.

13. Cheatham S.W., Stull K.R. (2018) Comparison of three different density type foam rollers on knee range of motion and pressure pain threshold: a randomized controlled trial. Int. J. Sports Phys. Ther., 13(3): 474-482. DOI: 10.26603/ijspt20180474.

14. Couture G., Karlik S., Glass S., Hatzel B. (2015) The effect of foam rolling duration on hamstring range of motion. Open Orthop. J., 9: 450-455.

15. Curran P., Flore R., Crisco J. (2008) A comparison of the pressure exerted on soft tissue by 2 myofascial rollers. J. Sport Rehabil., 17: 432-442.

16. D'Amico A., Paolone V. (2017) The effect of foam rolling on recovery between two eight hundred metre runs. J. Hum. Kinet., 57: 97-105.

17. DeBruyne D.M., Dewhurst M.M., Fischer K.M., Wojtanowski M.S., Dural C. (2017) Self-mobilization using a foam roller versus a roller massager: which is more effective for increasing hamstrings flexibility? J. Sport Rehabil., 26: 94-100.

18. Deguzman L., Flanagan S.P., Stecyk S., Montgomery M.M. (2018) The Immediate Effects of Self-administered Dynamic Warm-up, Proprioceptive Neuromuscular Facilitation, and Foam Rolling on Hamstring Tightness. Athletic Training \& Sports Health Care, 10(3): 108-116. DOI: 10.3928/19425864-20171101-07. 
19. Do K., Kim J., Yim J. (2018) Acute effect of self-myofascial release using a foam roller on the plantar fascia on hamstring and lumbar spine superficial back line flexibility. Phys. Ther. Rehabil. Sci., 7(1): 35-40.

20. Fairall R., Cabell L., Boergers R.J. Battaflia F. (2017) Acute effects of self-myofascial release and stretching in overhead athletes with GIRD. Journal of Bodywork \& Movement Therapies, 21: 648-652.

21. Findley F., Shelzola M. (2013) Fascia research congress evidence from the $100 \mathrm{y}$ perspective of Andrew Taylor Still.

22. Fleckenstein J., Wilke J., Vogt L., Banzer W. (2017) Preventive and regenerative foam rolling are equally effective in reducing fatigue-related impairments of muscle function following exercise. J. Sports Sci. Med., 16: 474-479.

23. Grabow L., Young J.D., Byrne J.M., Granacher U., Behm D.G., (2017) Unilateral Rolling of the Foot did not Affect Non-Local Range of Motion or Balance. J. Sports Sci. Med., 16: 209-218.

24. Griefahn A., Oehlmann J., Zalpour C., von Piekartz H. (2017) Do exercises with the foam roller have a shortterm impact on the thoracolumbar fascia? - A randomized controlled trial. J. Bodyw. Mov. Ther., 21: 186-193.

25. Grieve R., Clark J., Pearson E., Bullock S., Boyer C., Jarrett A. (2011) The immediate effect of soleus trigger point pressure release on restricted ankle joint dorsiflexion: a pilot randomised controlled trial. J. Bodyw. Mov. Ther., 15(1): 42-49.

26. Hall M., Smith J.C. (2018) The effects of an acute bout of foam rolling on hip range of motion on different tissues. Int. J. Sports Phys. Ther., 13(4): 652-660. DOI: 10.26603/ ijspt20180652.

27. Halperin I., Aboodarda S.J., Button D.C., Andersen L.L., Behm D.G. (2014) Roller massager improves range of motion of plantar flexor muscles without subsequent decreases in force parameters. Int. J. Sports Phys. Ther., 9(1): 92-102.

28. Hansen A., Beltz N.M., Janot J., Martenson A., Siegmann A., Jagielo A., Erdmann A., Wiggins, M. (2016) A dose response relationship between myofascial release and anaerobic power output in active college-aged males. Journal of Fitness Research, 5(2): 10-17.

29. Healey K.C., Hatfield D.L., Blanpied D., Dorfman L.R., Riebe D. (2014) The effects of myofascial release with foam rolling on performance. J. Strength Cond. Res., 28(1): 61-68.

30. Hinz B., Celetta G., Tomasek J.J., Gabbiani G., Chaponnier C.H. (2001) Alpha-smooth muscle actin expression upregulates fibroblast contractile activity. Mol. Biol. Cell., 12: 2730-2741.

31. Hodgson D., Lima C., Low J., Behm D. (2018) Four weeks of roller massage training did not impact range of motion, pain pressure threshold, voluntary contractile properties or jump performance. Inter. J. Sports Phys. Ther., 13(5): 835-845. DOI: 10.26603/ijspt20180835.

32. Hong C.Z., Simons D. (1998) Pathophysiologic and electrophysiologic mechanisms of myofascial trigger points. Arch. Phys. Med. Rehabil., 79: 863-872.

33. Jung J., Choi W., Lee Y., Kim J., Kim H., Lee K., Lee J., Lee S. (2017) Immediate effect of self-myofascial release on hamstring flexibility. Phys. Ther. Rehabil. Sci., 6(1): 45-51.

34. Kelly S., Beardsley C. (2016) Specific and cross-over effects of foam rolling on ankle dorsiflexion range of motion. Int. J. Sports Phys. Ther., 11(4): 544-550.

35. Lee C.L., Chu I.H., Lyu B.J., Chang W.D., Chang N.J. (2018) Comparison of vibration rolling, nonvibration rolling, and static stretching as a warm-up exercise on flexibility, joint proprioception, muscle strength, and balance in young adults. J. Sports Sci., 36(22): 2575-2582. DOI: 10.1080/02640414.2018.1469848.

36. MacDonald G.Z., Button D.C., Drinkwater E.J., Behm D.G. (2014) Foam rolling as a recovery tool after an intense bout of physical activity. Med. Sci. Sports Exerc., 131-42.

37. MacDonald G.Z., Penney M.D.H., Mullaley M.E., Cuconato A.L., Drake C.D.J., Behm D.G., Button D.C. (2013) An acute bout of self-myofascial release increases range of motion without a subsequent decrease in muscle activation or force. J. Strength Cond. Res., 27(3): 812-821.

38. Macgregor L.J., Fairweather M.M., Benett R.M., Hunter A.M. (2018) The effect of Foam Rolling for three consecutive days on muscular efficiency and range of motion. Sports Med., 4(26), DOI: 10.1186/s40798-018-0141-4.

39. Madoni S.N., Costa P.B., Coburn J.W., Galpin A.J. (2018) Effects of foam rolling on range of motion, peak torque, muscle activation, and the hamstrings-to-quadriceps strength ratios. J. Strength Cond. Res., 32(7): 1821-1830.

40. Maher C.G., Sherrington C., Herbert R.D., Moseley A.M., Elkins M. (2003) Reliability of the PEDro Scale for rating quality of randomized controlled trials. Phys. Ther., 83: 713-21.

41. Markovic G. (2015) Acute effects of instrument assisted soft tissue mobilization vs. foam rolling on knee and hip range of motion in soccer players. J. Bodyw. Mov. Ther., DOI: 10.1016/j.jbmt.2015.04.010.

42. Mauntel T.C., Clark M.A., Padua D.A. (2014) Effectiveness of myofascial release therapies on physical performance measurements: A systematic review. Athletic Training \& Sports Health Care, 6(4): 189-96.

43. McKenney K., Sinclair A., Elder C., Hutchins A. (2013) Myofascial release as a treatment for orthopaedic conditions: a systematic review. J. Athl. Train., 48(4): 522-527. 
44. Mohr A., Long B., Goad C. (2014) Effect of foam rolling and static stretching on passive hip-flexion range of motion. J. Sport Rehabil., 23: 296-299.

45. Monteiro E.R., Cavanaugh M.T., Frost D.M., Novaes J.S. (2017) Is self-massage an effective joint range-ofmotion strategy? A pilot study. J. Bodyw. Mov. Ther., 21: 223-226.

46. Monteiro E.R., Skarabot J., Vigotsky A.D., Brown A.F., Gomes T.M., Novaes J.S. (2017) Acute effects of different self-massage volumes on the FMS overhead deep squad performance. Int. J. Sports Phys. Ther., 12(1): 94-104.

47. Monteiro E.R., Skarabot J., Vigotsky A.D., Brown A.F., Gomes T.M., Novaes J.S. (2017) Maximum repetition performance after different antagonist foam rolling volumes in the inter-set rest period. Int. J. Sports Phys. Ther., 12(1): 76-84.

48. Monteiro E.R., Vigotsky A.D., Novaes J.S., Skarabot J. (2018) Acute effects of different anterior thigh selfmassage on hip range of motion in trained men. Int. J. Sports Phys. Ther., 13(1): 104-113. DOI: 10.26603/ijspt20180104.

49. Monteiro E.R., Vigotsky A.D., Skarabot J., Brown A.F., de Melo Fiuza A.G.F., Gomes T.M., Halperin I., da Silva Novaes J. (2017) Acute effects of different foam rolling volumes in the interset rest period on maximum repetition performance. Honk Kong Physiotherapy Journal, 36: 57-62.

50. Morton N.A. (2009) The PEDro scale is a valid measure of the methodological quality of clinical trials: a demographic study. Aust. J. Physiother., 55: 129-133.

51. Morton R.W., Oikawa S.Y., Phillips S.M., Devries M.C., Mitchell C.J. (2016) Self-myofascial release: no improvement of functional outcomes in ,tight" hamstrings. Int. J. Sports Physiol. Perform., 11: 658-664.

52. Moseley A.M., Herbert R.D., Sherrington C., Maher C.G. (2002) Evidence for physiotherapy practice: A survey of the Physiotherapy Evidence Database (PEDro). Aust. J. Physiother., 48: 43-49.

53. Murray A.M., Jones T.W., Horobenau C., Turner A.P., Sproule J. (2016) Sixty seconds of foam rolling does not affect functional flexibility or change muscle temperature in adolescent athletes. Int. J. Sports Phys. Ther., 11(5): 765-776.

54. Ozsu I., Gurol B., Kurt C. (2018) Comparison of the Effect of Passive and Active Recovery, and Self-Myofascial Release Exercises on Lactate Removal and Total Quality of Recovery. Journal of Education and Training Studies, 6 (9a): 33-42. DOI: 10.11114/jets.v6i9a.3511.

55. Patel D.G., Vyas N.J., Sheth M.S. (2016) Immediate effect of application of bilateral self myofascial release on the plantar surface of the foot on hamstring and lumbar spine: a quasi experimental study. IJTA, 32: 94-99.

56. Peacock C.A., Krein D.D., Silver T.A., Sanders G.J., Von Karlovitz K.P. (2014) An acute bout of self - myofascial release in the form of foam rolling improves performance testing. Int. J. Exerc. Sci., 7(3): 202-211.

57. Pearcey G.E.P., Bradbury-Squires D.J., Kawamoto J.E., Drinkwater E.J., Behm D.G., Button D.C. (2015) Foam rolling for delayed-onset muscle soreness and recovery of dynamic performance measures. J. Athl. Train, 50(1): 5-13.

58. Renan-Ordine R., Alburquerque-Sendin F., de Souza D.P.R., Cleland J.A., De-Las-Penas C.F. (2011) Effectiveness of myofascial trigger point manual therapy combined with a self-stretching protocol for the management of plantar heel pain: a randomized controlled trial. J. Orthop. Sports Phys. Ther., 41(2): 43-50.

59. Phillips J., Diggin D., King D.L., Sforzo G.A. (2018) Effect of varying self-myofascial release duration on subsequent athletic performance. J. Strength Cond. Res., Ahead of Print, DOI: 10.1519/JSC.0000000000002751.

60. Romero-Moraleda B., La Touche R., Lerma-Lara S., Ferrer-Pena R., Paredes V., Peinado A.B., Munoz-Garcia D. (2017) Neurodynamic mobilization and foam rolling improved delayed-onset muscle soreness in a healthy adult population: a randomized controlled trial. DOI: $10.7717 /$ peerj.3908.

61. Schroeder A.N., Best T.M. (2015) Is self myofascial release an effective preexercise and recovery strategy? A literature review. Curr. Sports Med. Rep., 4(3): 200-208.

62. Schroeder J., Renk V, Braumann K-M., Hollander K. (2017) Acute Foam Rolling effects on contractile properties of the m. biceps femoris. A randomized cross - over pilot study. Ger. J. Exerc. Sport Res., 47: 294-300. DOI: 10.1007/s12662-017-0467-y.

63. Simmonds N., Miller P., Gemmel H. (2012) A Theoretical Framework for the Role of Fascia in Manual Therapy. J. Bodyw. Mov. Ther., 16: 83-93.

64. Simons D.G. (2002) Understanding effective treatments of myofascial trigger points. J. Bodyw. Mov. Ther., 6(2): 81-88.

65. Skarabot J., Beardsley C., Stirn I. (2015) Comparing the effects of self-myofascial release with static stretching on ankle range-of-motion in adolescent athletes. Int. $J$. Sports Phys. Ther., 10(2): 203-212.

66. Stevens D. (2013) Foam rolling as a recovery aid for athletes. J. Aust. Strength Cond., 21(2): 43-51.

67. Stull K. (2016) Why should foam rolling be used in group training. American fitness, (4).

68. Sullivan K.M., Silvey D.B.J., Button D.C., Behm D.G. (2013) Roller-massage application to the hamstrings increases sit and reach ROM within 5 to 10 seconds without performance impairements. Int. J. Sports Phys. Ther., 8(3): 228-36.

69. Tatham J., Robergs R., Cameron M. (2016) The influence of self-myofascial release on countermovement jump force-time variables in pre-elite Academy Rubgy Union Players. J. Athl. Enhanc., 5(5), DOI: 10.4172/23249080.1000239. 
70. Van den Berg F. (2012) Macierz pozakomórkowa. In: Powięź. Badanie, profilaktyka i terapia dysfunkcji sieci powięziowej, red. Saulicz E., Wrocław, Elsevier Urban \& Partner, 197-203.

71. Vigotsky A.D., Lehman G.J., Contreras B., Beardsley C., Chung B., Feser E.H. (2015) Acute effects of anterior thigh foam rolling on hip angle, knee angle and rectus femoris length in the modified Thomas test. PeerJ, DOI: 10.7717/peerj.1281.

72. Weerapong P., Hume P.A., Kolt G.S. (2005) The mechanisms of massage and effects on performance, muscle recovery and injury prevention. Sports Med., 35(3): 235-256.

73. Wilke J., Vogt L. (2018) Immediate effects of self - myofascial release on latent trigger point sensitivity: a randomized, placebo - controlled trial. Biol. Sport, 35(4): 349-354.
74. Wines M.J. (2006) A complete meta analysis of myofascial release: application and efficacy for strength athletes. University of Carolina, EXSC 499.

75. Wiseman G. (2017) Effect of static stretching or foam rolling on hamstrings range of motion and strength.

\section{Received 29.06.2018}

Accepted 04.02.2019

(C) University of Physical Education, Warsaw, Poland

Acknowledgments

This work was supported by the Academy of Physical Education of Jerzy Kukuczka's, Katowice, Poland. 